\title{
Photon Dosimetry Using Plastic Scintillators in Pulsed Radiation Fields
}

\section{SPIE International Defense and Security Symposium}

\author{
David L. Chichester \\ Brandon W. Blackburn \\ James T. Johnson \\ Scott W. Watson
}

\section{April 2007}

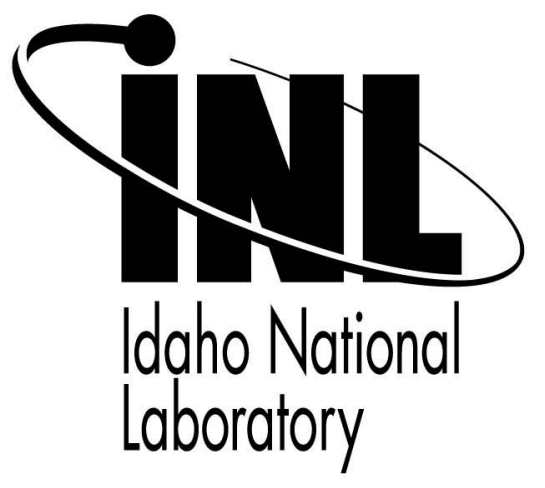

This is a preprint of a paper intended for publication in a journal or proceedings. Since changes may be made before publication, this preprint should not be cited or reproduced without permission of the author. This document was prepared as an account of work sponsored by an agency of the United States Government. Neither the United States Government nor any agency thereof, or any of their employees, makes any warranty, expressed or implied, or assumes any legal liability or responsibility for any third party's use, or the results of such use, of any information, apparatus, product or process disclosed in this report, or represents that its use by such third party would not infringe privately owned rights. The views expressed in this paper are not necessarily those of the United States Government or the sponsoring agency. 


\title{
Photon dosimetry using plastic scintillators in pulsed radiation fields
}

\author{
David L. Chichester*, Brandon W. Blackburn, James T. Johnson, and Scott W. Watson \\ Idaho National Laboratory, 2525 North Freemont Avenue, Idaho Falls, Idaho 83415
}

\begin{abstract}
Simulations and experiments have been carried out to explore using a plastic scintillator as a dosimetry probe in the vicinity of a pulsed bremsstrahlung source in the range 4 to $20 \mathrm{MeV}$. Taking advantage of the tissue-equivalent properties of this detector in conjunction with the use of a fast digital signal processor near real-time dosimetry was shown to be possible. The importance of accounting for a broad energy electron beam in bremsstrahlung production, and photon scattering and build-up, in correctly interpreting dosimetry results at long stand-off distances is highlighted by comparing real world experiments with ideal geometry simulations. Close agreement was found between absorbed energy calculations based upon spectroscopic techniques and calculations based upon signal integration, showing a ratio between $10 \mathrm{MeV}$ absorbed dose to $12 \mathrm{MeV}$ absorbed dose of 0.58 at a distance of $91.4 \mathrm{~m}$ from the accelerator. This is compared with an idealized model simulation with a monoenergetic electron beam and without scattering, where the ratio was 0.46 .
\end{abstract}

Keywords: Plastic scintillator, bremsstrahlung, dosimetry, pulsed radiation

\section{INTRODUCTION}

Next generation cargo screening systems currently under development will use energetic x-rays to generate high resolution images of shipping containers, to detect the presence of high density objects and to identify the presence of special nuclear material (SNM) including uranium and plutonium. The images from these systems will be used to perform general verification of cargo manifests and localize dense objects within the containers. The presence of fissile material may be determined by monitoring the cargo for emission signatures from photofission of photonuclear resonance excitation. In most cases the x-rays used in these devices will be generated using high power electron accelerators and will usually be operated in pulsed modes. In addition, high energy photonuclear active interrogation systems are also being developed for stand-off detection systems with goals of identifying the presence of shielded SNM at distances of greater than 5 meters from the radiation source. In these cases larger and more powerful probe radiation sources will be used.

One challenge facing both cargo screening systems and stand-off interrogation systems is in the area of radiation health physics instrumentation. Using high energy bremsstrahlung of $10 \mathrm{MeV}$ or greater these systems will generate extremely intense radiation fields which in open configurations may extend up to several hundred meters from the accelerators. The dose field will depend upon electron energy and beam current, choice of converter target and collimation, the presence of nearby scattering objects, and any shielding which may be present. Mapping of these radiation fields will also be complicated by the need to use pulsed x-ray bursts in most screening approaches. Most commonly available commercial electron accelerators operate at frequencies from $10-500 \mathrm{~Hz}$ with pulse widths of 10 microseconds or less. These operating parameters are desirable both for collecting high resolution imagery and for photofission interrogation when delayed photon or neutron radiation signatures are being examined. Unfortunately, high intensity short-burst pulsed radiation is difficult to analyze using standard health physics instrumentation which is primarily intended for use in steady-state environments.

Idaho National Laboratory in collaboration with Idaho State University is currently researching large scale security screening technologies at the Inspections Technology Research and Development Laboratory (ITRDL) test bed in Pocatello, Idaho. Research at ITRDL includes both cargo screening and stand-off interrogation projects which use variable energy electron accelerators capable of operating up to $25 \mathrm{MeV}$. When operating at their full energy the radiation fields used for long-range stand-off detection can extend several hundred meters from the accelerator's

* David.Chichester@inl.gov; phone +1 208 526-8920; fax +1 208 526-6239; www.inl.gov 
bremsstrahlung converter plate. Measuring site boundary radiation levels, in the 100 microrem range, and verifying compliance with applicable health physics safety regulations is difficult using standard hand-held instruments since all operations at this facility are pulsed. Due to the relatively low sensitivity of these devices accelerator operations must be maintained for several days to conduct a complete area survey and determine compliance with the site's boundary radiation regulatory levels. This process is time consuming and costly, especially since repetitive surveys are often necessary due to changes in the research program or facility shielding layout. In response to these challenges research is underway at INL to develop real-time analytical instruments capable of monitoring pulsed field photon dose rates.

Plastic scintillators have been extensively used in medical health physics dosimetry as in-vivo probes during radiation treatment and therapy; excellent references on the subject are provided by Belcher, O'Foghludha, and Beddar. ${ }^{1-3}$ Dosimetry data may be collected with a scintillator in several different ways. For example, the output current from the device, which is directly proportional to the energy deposited in the scintillator, can be recorded and integrated over a period of time to determine dose according to (1). At high dose rates when the output current is a continuum this can be done using an ammeter; at lower dose rates, when energy deposition results in discrete charge pulses, fast pulse digitization and signal processing becomes useful to maintain measurement precision.

$$
\begin{gathered}
\text { Dose }=\frac{\text { Deposited Energy }}{\text { Mass of Scintillator }} \\
\text { Deposited Energy }=\int_{0}^{E_{\max }} \mathrm{E} \cdot \mathrm{N}(\mathrm{E}) \mathrm{dE}
\end{gathered}
$$

Alternatively, the absorbed dose may be determined by using standard spectroscopic techniques generating a pulse height histogram that represents energy deposition into the scintillator. With this histogram, one technique is to calculate an energy deposition spectrum (multiplying each bin by it's average energy) and then integrating the spectrum to calculate the dose according to (1) and (2). Another approach for dose determination using a pulse height histogram described by Lobdell and Hertel involves unfolding the pulse height spectrum to infer the actual photon flux distribution irradiating the scintillator, then convolving this information with energy dependent flux-to-dose conversion factors to determine dose. ${ }^{4,5}$ A complication to this approach is the need to develop a radiation transport model for the detector to unfold the raw data and determine the original irradiation spectrum profile.

Plastic scintillators are well-suited for direct measurements of photon dose due to their close similarity in both density and chemical composition with human tissue. This is the primary reason why plastic scintillators have been so extensively studied for use as in-situ real-time dosimetry probes for high-energy bremsstrahlung medical radiation treatment monitoring. ${ }^{1,6-10}$ Medical dosimetry research has shown common plastic scintillators to have water equivalent (tissue equivalent) energy deposition properties from 0.2 to $20 \mathrm{MeV}$, with the ratio of dose deposited in plastic to that

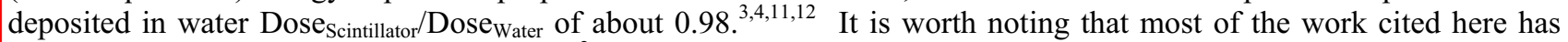
involved placing small scintillators $\left(<1 \mathrm{~cm}^{2}\right)$ within the body or a water-equivalent phantom, therefore establishing a charge particle equilibrium (CPE) condition that satisfies the Bragg-Gray principle. ${ }^{1,13}$ One exception to these medical applications was in the use of a larger plastic scintillator $(2.54 \times 2.54 \mathrm{~cm}$ cylinder, BC 402) for area radiation surveys in a nuclear power plant environment. ${ }^{4}$ Although the authors of this work did not specifically comment on the establishment of charged particle equilibrium it probably did exist due to the use of a plastic shroud intended to attenuate stray background electron signals from reaching their detector. Without charged particle equilibrium relative estimates of dose profiles can still be made for bremsstrahlung spectrum with similar end-point energies but absolute calibration over a wide range based becomes more challenging.

In this paper we present preliminary scoping work performed at INL to examine the use of plastic scintillators for low intensity high-energy photon dosimetry in pulsed radiation beams. The eventual goal of this work is to develop a handheld device for performing real-time area survey measurements in the pulsed radiation fields from active interrogation security screening systems. In the following sections we present information on simulations performed to estimate the response signature for a plastic scintillator located $100 \mathrm{~m}$ from bremsstrahlung sources of 4-20 MeV, and experimental results from measurements taken $100 \mathrm{~m}$ from $10 \mathrm{MeV}$ and $12 \mathrm{MeV}$ bremsstrahlung exposure. 


\section{SIMULATION AND MODELING}

Modeling was performed to generate a set of generic bremsstrahlung radiation profiles from $4-20 \mathrm{MeV}$ using the MCNP5 radiation transport simulation code. ${ }^{14}$ For these simple models monoenergetic electron were simulated impinging upon a $1 \mathrm{~mm}$ thick tungsten converter plate, which is representative of converter options used at the ITRDL facility (the optimal converter thickness for dose maximization in $\mathrm{W}$ bremsstrahlung converter targets ranges from 0.3 to $0.95 \mathrm{~mm}$ over the 4 to $20 \mathrm{MeV}$ range). ${ }^{15}$ Photon flux was calculated on-axis with the electron beam, $1 \mathrm{~m}$ from the converter plate in $1 \mathrm{keV}$ energy bins. To save on computing time, the intensity in each bin of these spectra was then attenuated according to (3) to generate approximate on-axis spectra corresponding to the transport of the original spectra though $100 \mathrm{~m}$ of dry air, using attenuation data from Hubbel. ${ }^{16}$

$$
I=I_{0} \exp (-\mu \cdot d)
$$

These $100 \mathrm{~m}$ photon spectra were then used as the input for a second simulation using MCNP5 which modeled the energy deposition and interaction histogram results in a plastic scintillator. For this simulation the detector had a standard plastic composition and cylindrical geometry with a diameter of $2 \mathrm{~cm}$ and a length of $10 \mathrm{~cm}$. In this case the radiation source was modeled as a planar source $2 \mathrm{~cm} \times 10 \mathrm{~cm}$ aligned with the geometry of the scintillator, emitting photons normal to its surface and $10 \mathrm{~cm}$ away from the scintillator (see Fig. 1). (Note: This arrangement is similar to that used to collect field data as described in below in that neither probe included an external shroud to establish CPE. Because of this the results are presented in a relative scale and have not been extrapolated to units of dose in either case.) Both photon and electron transport was modeled, with electron and photon energy cut-off levels of $1 \mathrm{keV}$.

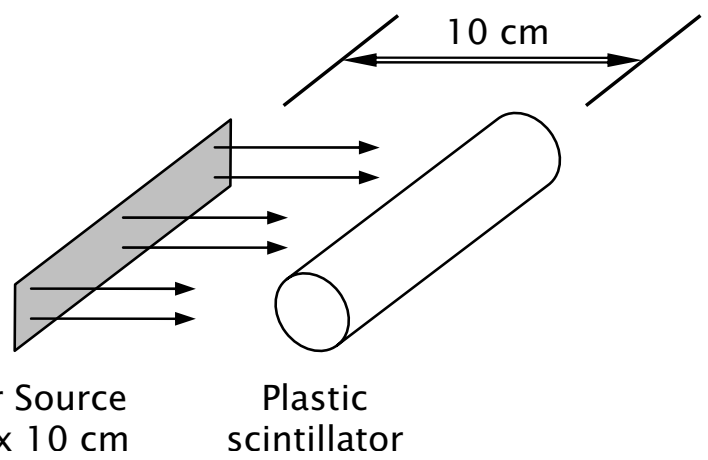

Fig. 1 Schematic representation of the geometry used to model irradiation of a generic plastic scintillator using a $100 \mathrm{~m}$ extrapolated bremsstrahlung beam. 

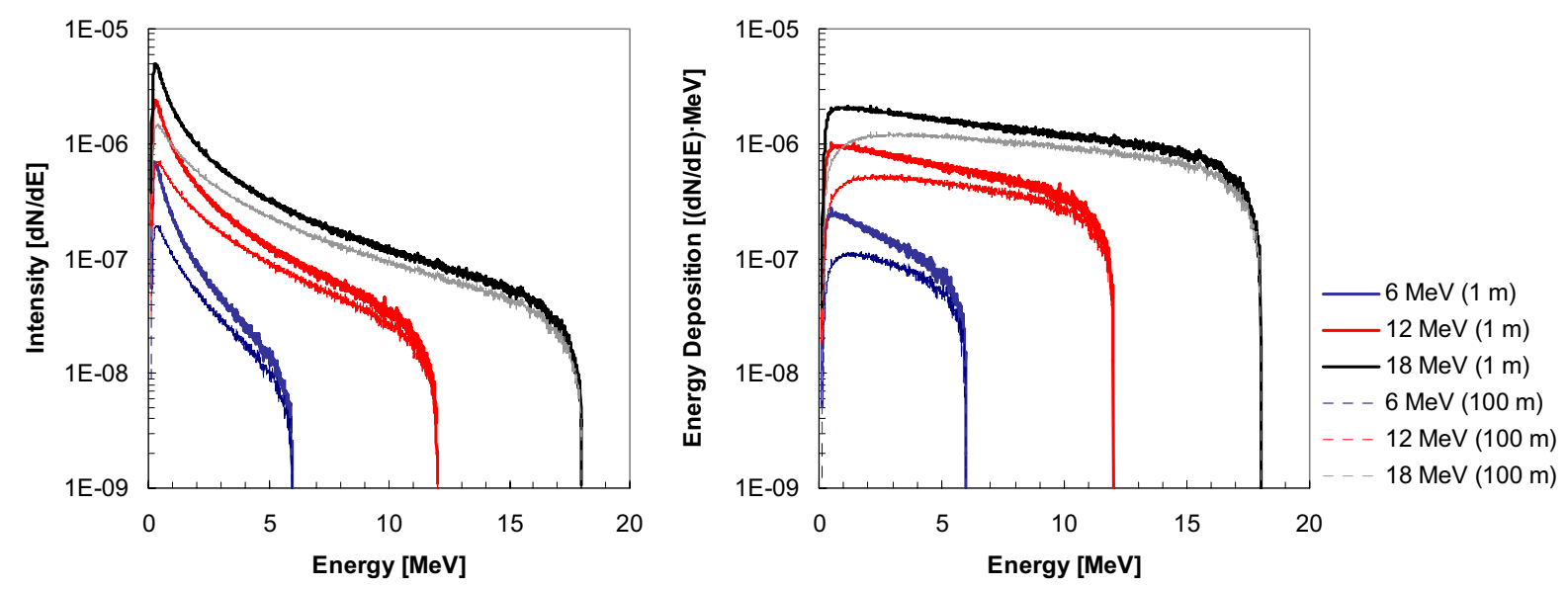

Fig. 2 Spectral shape of bremsstrahlung $1 \mathrm{~m}$ from a $1 \mathrm{~mm}$ tungsten converter (thick lines) and the extrapolated spectrum after attenuation through $100 \mathrm{~m}$ of air (thin lines) on the left and corresponding spectral shape of energy weighted spectra on the right.

The calculated 1 meter and $100 \mathrm{~m}$ bremsstrahlung spectra from 6,12, and $18 \mathrm{MeV}$ electrons are shown in Fig. 2 (left) together with the energy weighted values for these spectra (calculated by multiplying each bin value with the average energy of that bin as described in ref. 4) (right). Due to attenuation through $100 \mathrm{~m}$ of air, this idealized radiation field becomes somewhat harder than seen at the 1 meter location. (As discussed in the measurement sections below this is an idealized situation, scattered radiation probably adds a significantly to the lower energy regions of the actual spectrum.) The relative energy in the radiation field at $1 \mathrm{~m}$ and $100 \mathrm{~m}$ is shown in Fig. 3 where it has been normalized to the case for a $12 \mathrm{MeV}$ end point beam energy at $1 \mathrm{~m}$, showing that the relative energy in the beams between 4 and $20 \mathrm{MeV}$ (neglecting low energy build-up due to scattering) is similar between $1 \mathrm{~m}$ and $100 \mathrm{~m}$.

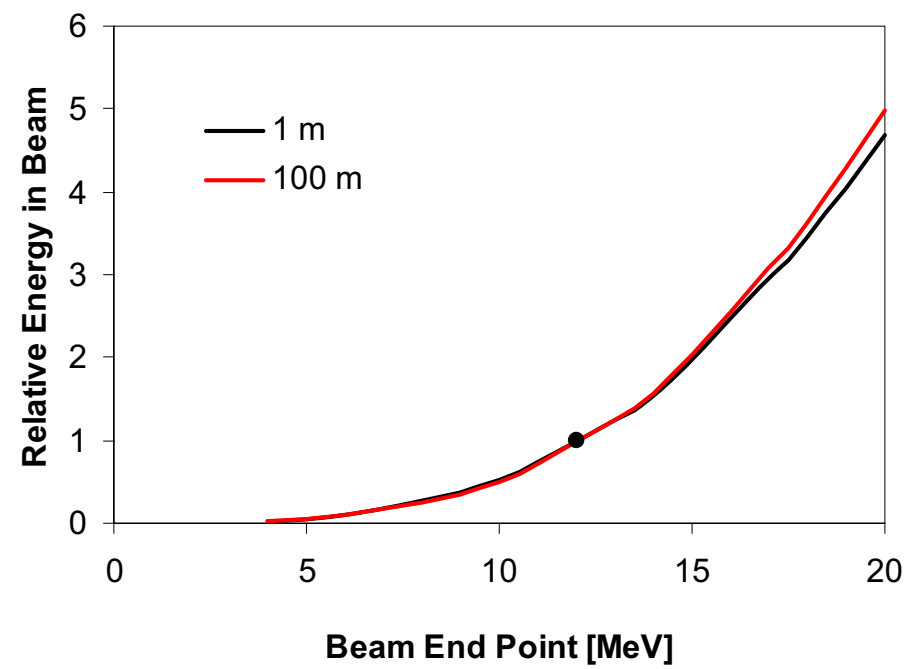

Fig. 3 Relative energy in the bremsstrahlung beam for 4 through $20 \mathrm{MeV}$ end point energies at $1 \mathrm{~m}$ and $100 \mathrm{~m}$ showing effects of attenuation but neglecting likely low energy build-up due to scattering. 

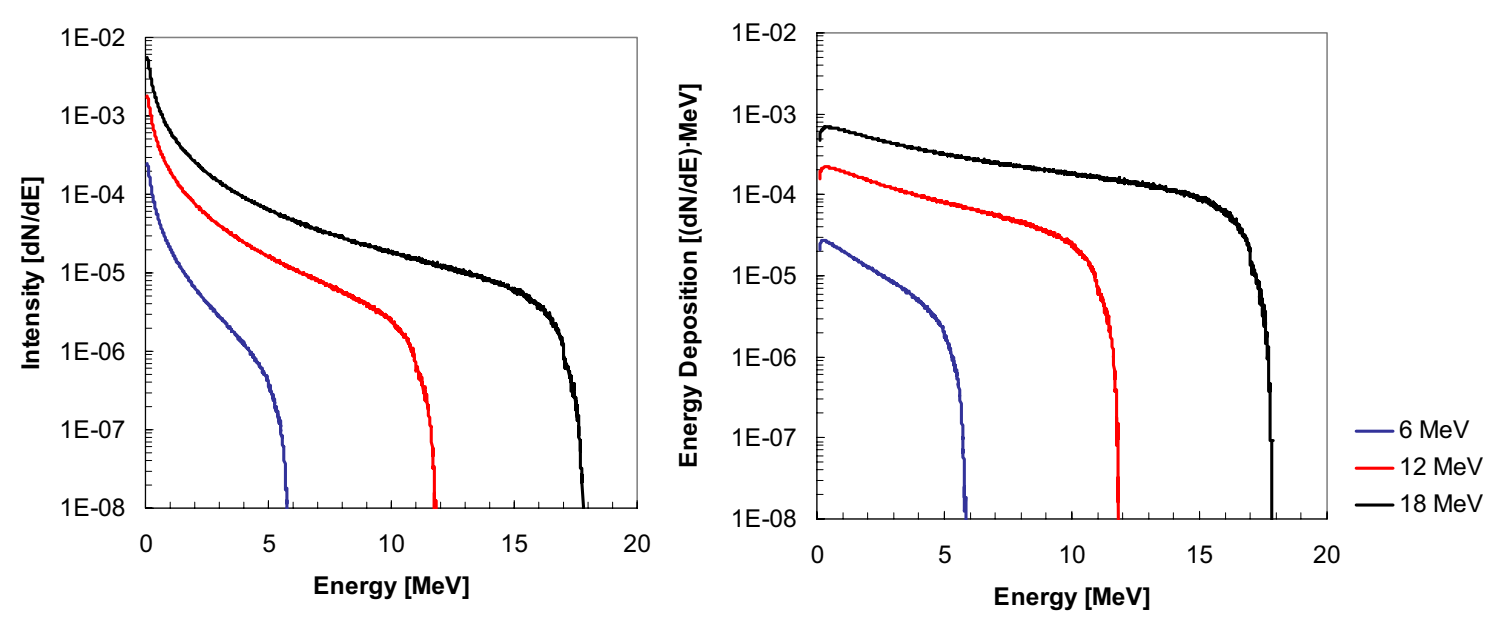

Fig. 4 Recoil spectra of energy events in the plastic scintillator $100 \mathrm{~m}$ from the bremsstrahlung source (left) and energy deposition spectra (right) for 6,12 , and $18 \mathrm{MeV}$ end-point energies.

In Fig. 4 the event spectrum in the plastic scintillator is shown (left), which is essentially a plot of electron recoil events in the plastic scintillator resulting from Compton scattering and pair production and the subsequent electron interactions in the plastic. This data has been converted to an energy deposition spectrum within the detector on the right side of Fig. 4. If the energy deposition spectra bins are summed together an estimate can be made of the relative energy (dose) deposited in the plastic scintillator for different beam energies; this is shown in Fig. 5. In the experiments described below exposure measurements were taken with $10 \mathrm{MeV}$ and $12 \mathrm{MeV}$ bremsstrahlung beams. From Fig. 5 the ratios of absorbed dose from a $10 \mathrm{MeV}$ beam to absorbed dose from a $12 \mathrm{MeV}$ beam with $0,1,2$, and $3 \mathrm{MeV}$ threshold levels are $0.46,0.43,0.41$, and 0.38 , respectively.

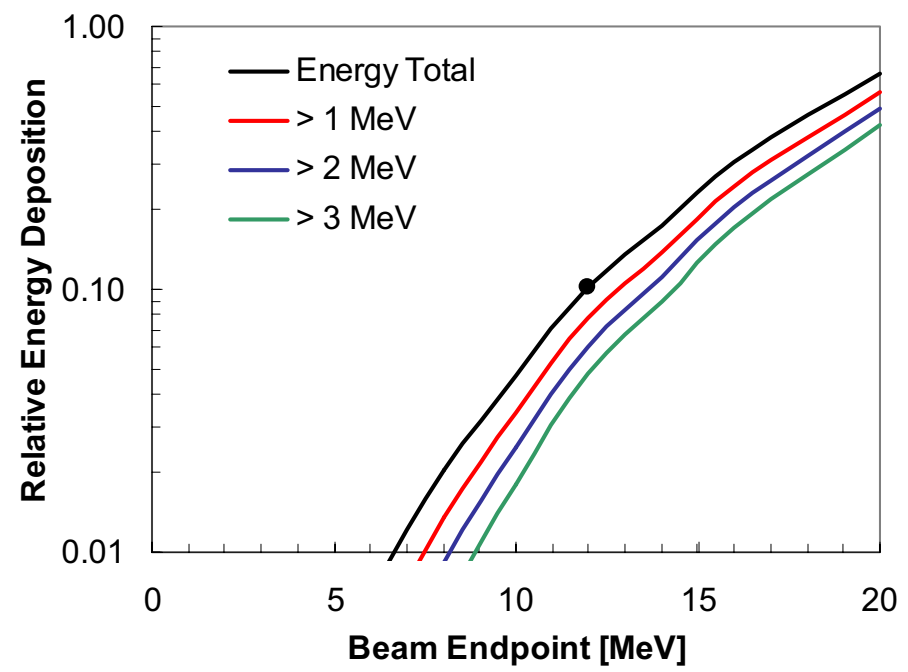

Fig. 5 Idealized recoil spectra of energy events in the plastic scintillator $100 \mathrm{~m}$ from the bremsstrahlung source (left) and energy deposition spectra (right) for 6, 12, and $18 \mathrm{MeV}$ end-point energies.

\section{EXPERIMENTAL TECHNIQUE}

To investigate the use of a plastic scintillator for pulsed dosimetry measurements experiments were performed at the INL/ISU ITRDL facility using an EJ-200 plastic scintillator (equivalent to Pilot $F$ and BC 408) from Eljen Technology. ${ }^{17}$ The detector was rectangular with dimensions of $2.54 \mathrm{~cm} \times 2.54 \mathrm{~cm} \times 10.16 \mathrm{~cm}$ and was coupled to a 
Hamamatsu R6095-03 photomultiplier operated at $-700 \mathrm{~V}$. The detector was placed $91 \pm 1 \mathrm{~m}$ from the converter plate (tungsten, $2 \mathrm{~mm}$ thick) of a multi-energy electron linear accelerator. Lead shielding was located in the immediate vicinity of the converter to provide a $0.62 \mathrm{~cm}$ square pencil beam collimation. Concrete shield walls were also on the sides and behind the accelerator. The collimated x-ray beam projected from the three-walled concrete enclosure to an outdoor environment with a concrete pad. The accelerator was operated at 10 and $12 \mathrm{MeV}$, beam current was monitored using a Faraday cup on the accelerator $( \pm 5 \%)$.. The accelerator was operated at a frequency of $125 \mathrm{~Hz}$ with a pulse width of approximately $\sim 3$ microseconds.

The output from the detector was sent directly to a DC282 high-speed digitizer from Agilent Technologies. ${ }^{18}$ This 10-bit digitizer was used to collect and store waveforms from the scintillator in $0.5 \mathrm{~ns}$ time bins. An external trigger signal from the accelerator was used to start data collection in the digitize. An in-house computer program was used to process data from these experiments. Data transfer from the digitizer to the computer was a limiting factor, processed waveforms were captured at approximately $35 \mathrm{~Hz}$, resulting in collection of $28 \%$ of the available signal. For both the 10 $\mathrm{MeV}$ and $12 \mathrm{MeV}$ cases 20,000 waveforms were captured. An example of one complete $8 \mu$ s digitized waveform from the $10 \mathrm{MeV}$ test is shown in Fig. 6. A small DC offset was present on the scintillator's signal, as seen in the highlighted region from $0.5-1.0 \mu \mathrm{s}$ in Fig. 6 of $1.46 \mathrm{mV} / \mathrm{bin}$; when integrated over the entire waveform this offset was $23.4 \mathrm{~V}$ per 8 $\mu \mathrm{s}$.
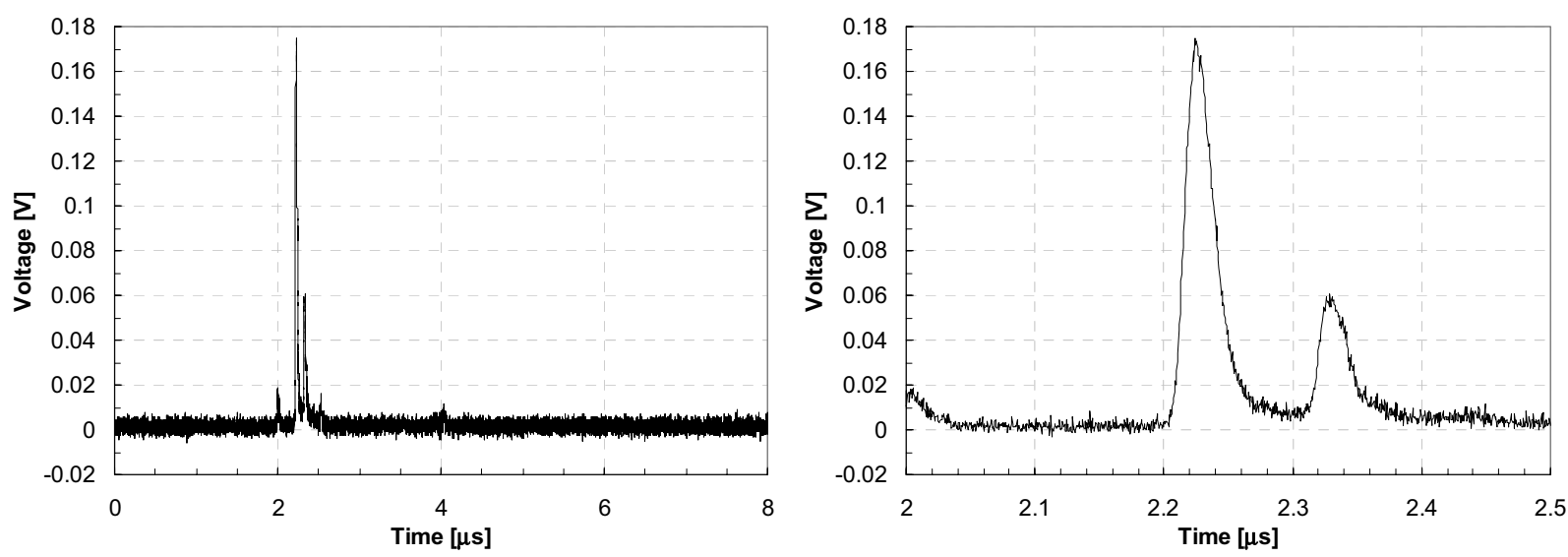

Fig. 6 Example of a complete scintillator pulse waveform (left) and a close-up plot of the same waveform from 3.5 to 4.0 $\mu \mathrm{s}$ showing two individual pulses (right). The section from $0.5-1.0 \mu \mathrm{s}$ is representative of the background signal.

\section{RESULTS}

Twenty-thousand waveforms were captured while operating at $10 \mathrm{MeV}$ and at $12 \mathrm{MeV}$, two methods were used to convert this information into a form representative of energy absorbed in the scintillator. Section 4.1 outlines the process used to develop a rough energy calibration for electron recoil events in the scintillator. In section 4.2 a pulse height histogram is developed from the data and used to calculate absorbed dose; in section 4.3 the integral voltage value of each waveform is used to determine absorbed energy.

\subsection{Rough Energy Calibration}

Prior to collecting bremsstrahlung spectral data the detector was exposed to radiation from a combined Cs-137 and Co60 check source. The Cs- 137 emits photons at $0.6617 \mathrm{MeV}$ while the Co-60 emits two photons of equal intensity at 1.1732 MeV and $1.3325 \mathrm{MeV}$. Compton scattering is the primary interaction mechanism for these photons in the scintillator and results in a distribution of electron recoil energy being deposited in the detector. A maximum value of scattered energy that can be deposited in the detector from single interactions exists which can be determined according to (4) where $E \gamma$ is the decay photon energy and $\mathrm{m}_{0} \mathrm{c}^{2}$ is the rest mass energy of the electron $(0.511 \mathrm{MeV})$. For the three photon energies above, the maximum Compton photon energy $\mathrm{E}_{\max }$ is $0.4767 \mathrm{MeV}, 0.9634 \mathrm{MeV}$, and $1.1181 \mathrm{MeV}$, respectively. 


$$
\mathrm{E}_{\max }=\mathrm{E}_{\gamma}-\mathrm{E}_{\mathrm{C}}=\mathrm{E}_{\gamma}-\frac{\mathrm{E}_{\gamma}}{1+2 \mathrm{E}_{\gamma} / \mathrm{m}_{0} \mathrm{c}^{2}}
$$

The calibration spectra is shown in Fig. 7. A simple energy calibration was been developed by averaging the two Co-60 Compton edge values and placing this at $70 \%$ of the maximum intensity of the Compton plateau from these interactions and doing the same for the Cs-137 part of the spectrum (corrected for the underlying Co-60 background) according to the general guideline suggested in ref. 4. A more precise calibration could be developed by modeling the detectors performance as suggested in this reference and by Kudomi but this was not justified for the scoping experiments presented here. ${ }^{19}$

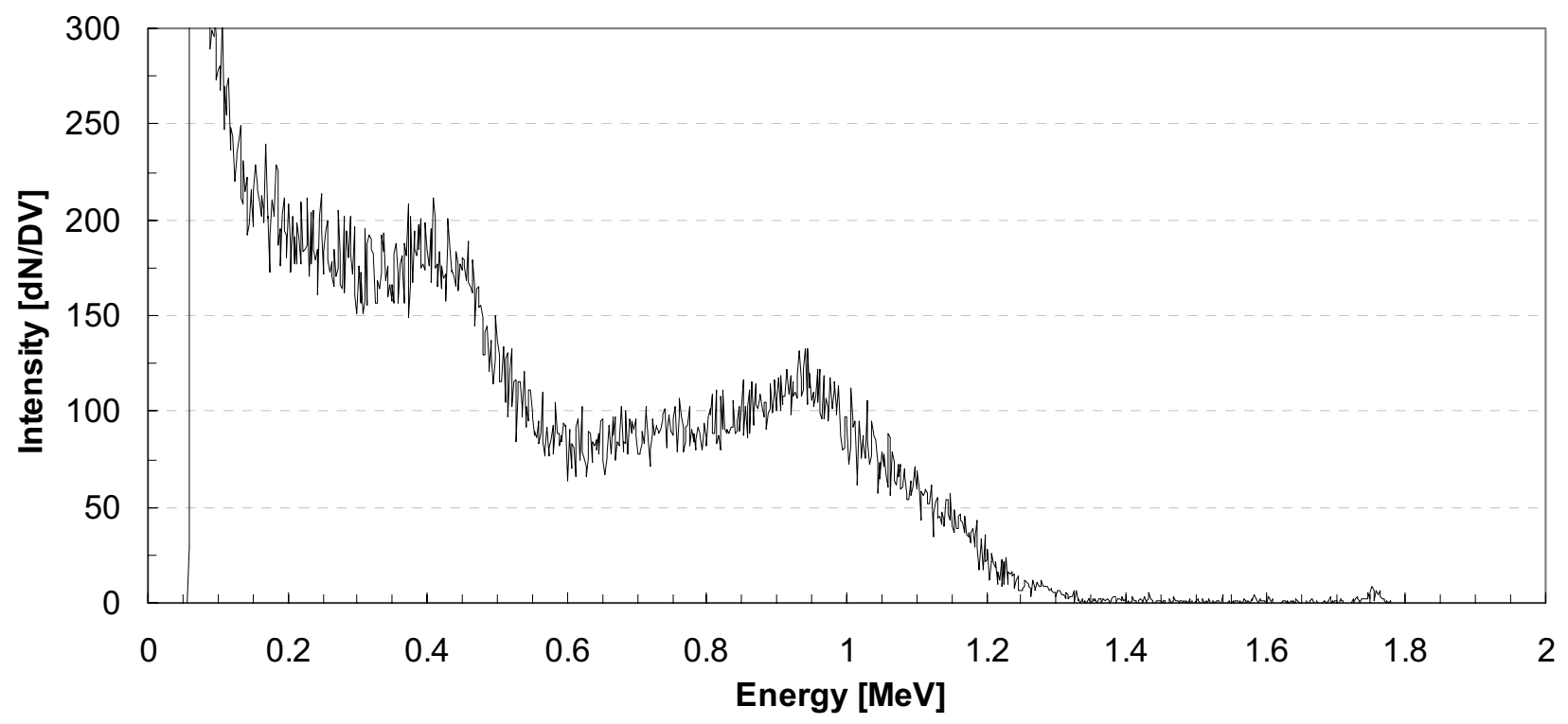

Fig. 7 Scintillator response to combined exposure from Cs-137 and Co-60.

\subsection{Pulse Height Analysis}

A pulse height histogram, using the energy calibration derived above, for the data collected during the $10 \mathrm{MeV}$ and 12 $\mathrm{MeV}$ runs is shown in Fig. 8. A clear distinction can be seen between the $10 \mathrm{MeV}$ and $12 \mathrm{MeV}$ cases although, in comparing this figure with the energy deposition spectra calculated using MCNP, both spectra contain significantly more lower-energy events resulting in a softer beam spectrum (the slope of the spectra is steeper in Fig. 8 than in Fig. 4). 


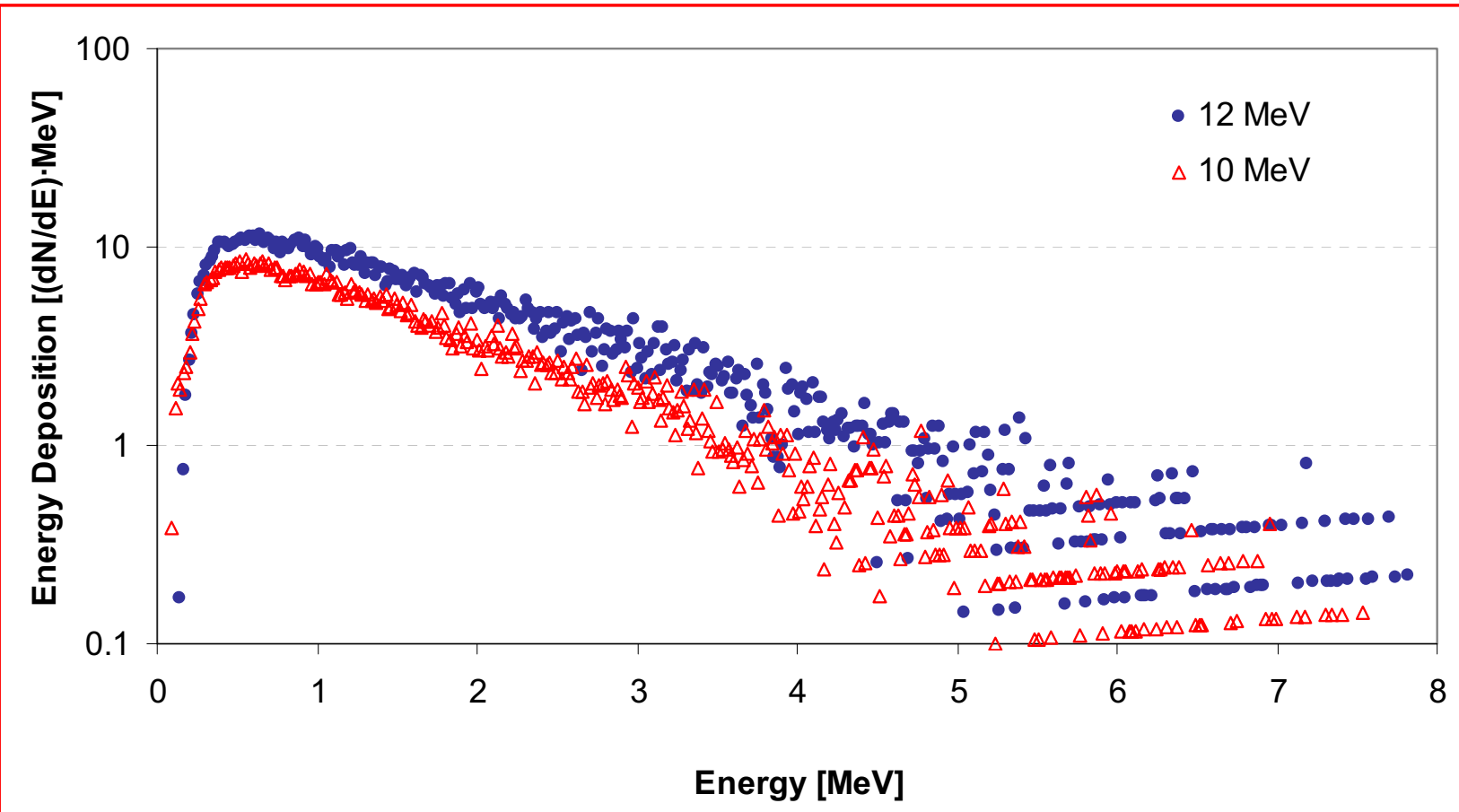

Fig. 8 Plastic scintillator pulse height spectra at $10 \mathrm{MeV}$ and $12 \mathrm{MeV}$ (normalized to account for different average accelerator beam currents for each run).

This is probably due to photon scattering near the accelerator in the lead collimator, photon scattering in the atmosphere and off the ground as the beam transits the $91.4 \mathrm{~m}$ distance between the accelerator and the detector, and contamination in the detector exposure from background delta rays (free electrons) generated due to Compton scattering of these same photons in the air near the detector - all of which skew the unaltered beam by producing more lower-energy photons. Because of the asymmetric presence of these lower energy scattered photons and electrons the difference in absorbed energy for the two beam energies calculated by summing together all of the bins in each of the two spectra in Fig. 8 is not as large as predicted from the simple simulation model described above. Also, in the model work ideal monoenergetic electron beams were simulated while in practice the accelerator energy beam is spread in energy with a FWHM of roughly $1 \mathrm{MeV}$, an effect which would also serve to reduce the difference in absorbed dose between the 10 and $12 \mathrm{MeV}$ cases. For this analysis the ratio of absorbed energy $10 \mathrm{MeV} / 12 \mathrm{MeV}$ for all events is $0.58 \pm 0.12$ versus 0.46 for the idealized case presented in the modeling.

\subsection{Complete Waveform Integration}

Using the same data, a complete integration of the voltage value in each of the 16,000 bins in each of the 20,000 waveforms has also been calculated, as an alternative method of determining absorbed energy in the scintillator. The results are presented in Fig. 9, where each of the waveform integrated voltage values is shown as a histogram of number of waveforms per $0.156 \mathrm{~V}$ energy bin. For the $10 \mathrm{MeV}$ case $828(4.1 \%)$ of the waveforms didn't contain distinct pulses, at $12 \mathrm{MeV}$ this total was $854(4.3 \%)$, none of these waveforms were included in the histograms. It is clear in reviewing these spectra that the DC offset voltage level mentioned above (Fig. 6) changed between the $10 \mathrm{MeV}$ and $12 \mathrm{MeV}$ runs.. To account for this effect each of the two curves in Fig. 9 was fitted to extrapolate the background DC offset voltage. For the $10 \mathrm{MeV}$ data the $\mathrm{DC}$ bias value was found to be $23 \mathrm{~V}$, for the $12 \mathrm{MeV}$ case the value was $11.5 \mathrm{~V}$. Accounting for this bias, and correcting for different average beam currents between the two runs, the ratio of absorbed energy 10 $\mathrm{MeV} / 12 \mathrm{MeV}$ determined using this approach was $0.58 \pm 0.10$ versus 0.46 for the idealized case presented in the modeling. 


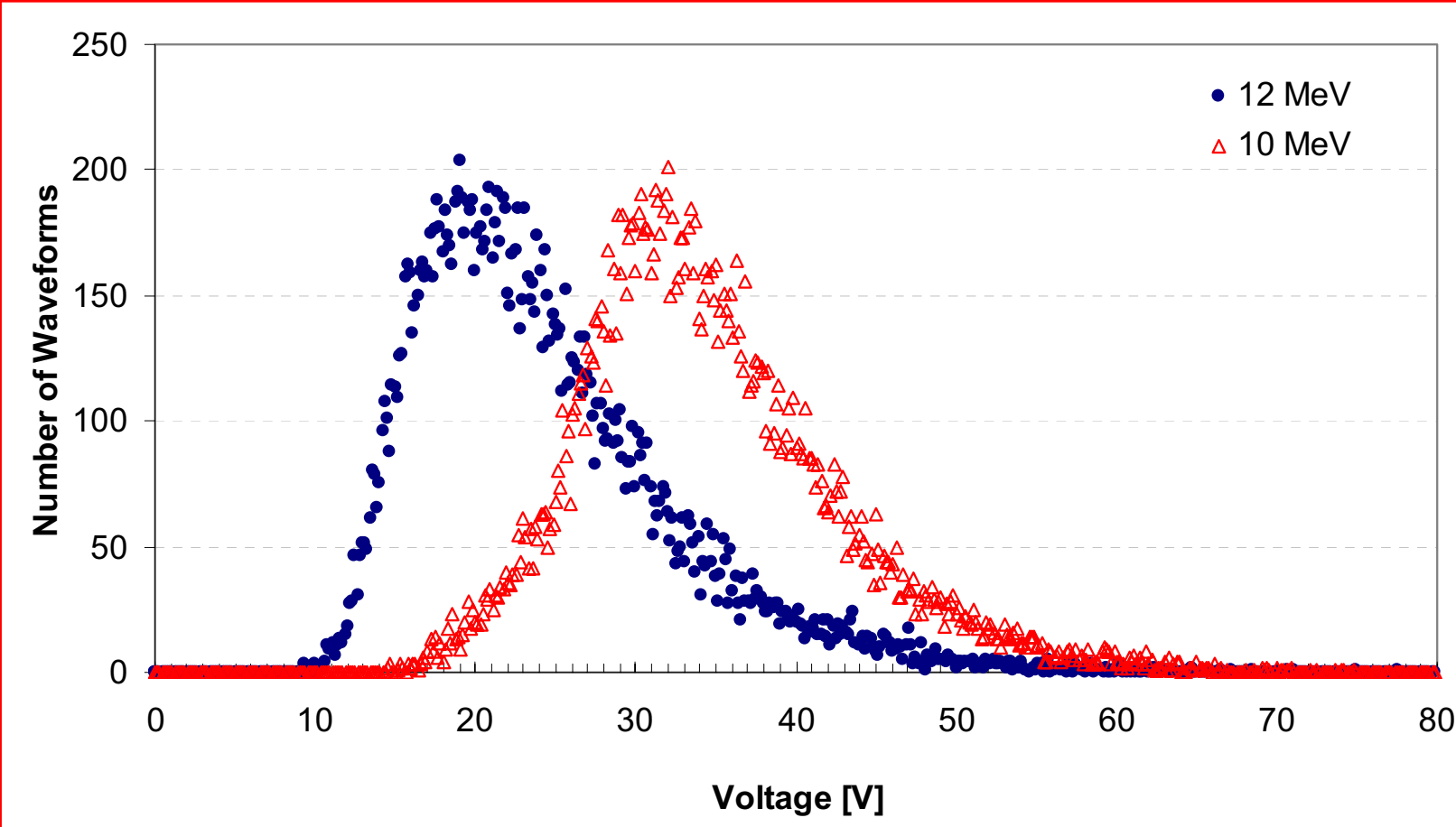

Fig. 9 Integrated waveform voltage histograms at $10 \mathrm{MeV}$ and $12 \mathrm{MeV}$.

\section{DISCUSSION}

Modeling and experimental work has demonstrated the feasibility of using a tissue-equivalent scintillator to perform near real-time dosimetry in the vicinity of pulsed high-energy bremsstrahlung. Previous research in the area has shown the validity of the technique as used in the medical health physics field with bremsstrahlung and has also been demonstrated in the industrial setting of a nuclear power plant with monoenergetic high energy photons. This work has shown the extension of this technique for measurements in pulsed radiation fields. Using a high speed digitizer this work has shown how absorbed energy in a plastic scintillator can be determined using either standard spectroscopic techniques through the calculation of an absorbed energy spectrum or, more simply, by performing a simple integration of the output signal from a plastic scintillator. Excellent agreement was found between these two simple exposure measurements at $10 \mathrm{MeV}$ and $12 \mathrm{MeV}$ with an average $\mathrm{D}_{10 \mathrm{MeV}} / \mathrm{D}_{12 \mathrm{MeV}}$ ratio of 0.58 . In comparison, a simple simulation of an idealized geometry, which included a monoenergetic electron source and neither photon scattering from a beam collimator nor the effects of photon scattering in the atmosphere in transit from the accelerator to the detector, produced a $\mathrm{D}_{10 \mathrm{MeV}} / \mathrm{D}_{12 \mathrm{MeV}}$ ratio of 0.46 .

Future improvements planned for this technique includes i) the use of a CPE shroud, ii) using an electromagnetic shield to protect the detector PMT, iii) identifying the cause of the non-zero, variable DC bias offset and eliminating it, iv) developing an MCNP detector model for the scintillator to allow spectra unfolding and more precise energy calibrations, v) developing a more realistic MCNP simulation of the entire process including a broad energy electron beam and photon scattering over the $100 \mathrm{~m}$ transit, vi) performing additional experiments and more energies with co-located TLD dosimeters for benchmark testing, vii) extrapolation of this technique for use with liquid scintillators to allow photonneutron discrimination, viii) the development of algorithms for performing simultaneous photon and neutron dosimetry, ix) performing laboratory evaluations of the device using dosimetry reference standards available at INL's Health Physics Instrumentation laboratory, and $\mathrm{x}$ ) exploring the use of larger dosimeters to increase the measurement sensitivity and reduce data acquisition times. ${ }^{20}$ 


\section{REFERENCES}

1. Belcher, E. H., "Radiation dosimetry with scintillation detectors," Brit. J. Radiology 26, 455-464 (1953).

2. O'Foghludha, F., "Discussion: Scintillation dosimetry, and an application of it," Annals N.Y. Acad. Sci.161, 86-96 (1969).

3. Beddar, A. S., Mackie, T. R., and Attix, F. H, "Water-equivalent plastic scintillation detectors for high-energy beam dosimetry: I. Physical characteristics and theoretical considerations," Phys. Med. Biol. 37, 1883-1900 (1992).

4. Lobdell, J. L. and Hertel, N. E., "Photon spectra and dose measurements using a tissue-equivalent plastic scintillator," Rad. Prot. Dos. 72, 95-103 (1997).

5. "Neutron and gamma-ray fluence-to-dose factors," ANSI/ANS-6.1.1-1991, American Nuclear Society, La Grange Park, IL (1991).

6. Beddar, A. S., Mackie, T. R., and Attix, F. H, "Water-equivalent plastic scintillation detectors for high-energy beam dosimetry: II. Properties and measurements," Phys. Med. Biol. 37, 1901-1913 (1992).

7. Flühs, D., Heintz, M., Indenkämpen, and Wieczorek, C., "Direct reading measurement of absorbed dose with plastic scintillators - The general concept and application to ophthalmic plaque dosimetry," Med. Phys. 23, 427434 (1996).

8. Beddar, A. S., Kinsella, T. J., Ikhlef, A., and Sibata, C. H., "A miniature "scintillator-fiberoptic-PMT" detector system for the dosimetry of small fields in stereotactic radiosurgery," IEEE Trans. Nucl. Sci. 48, 924-928 (2001).

9. Petric, M. P., Robar, J. L., and Clark, B. G., "Development and characterization of a tissue equivalent plastic scintillator based dosimetry system," Med. Phys. 33, 96-105 (2006).

10. Beddar, A. S., "Water equivalent plastic scintillation detectors in radiation therapy," Rad. Prot. Dos. 120, 1-6 (2006).

11. Beddar, A. S., Briere, T. M., Mourtada, F. A., Vassiliev, O. N., Liu, H. H., and Mohan, R., "Monte Carlo calculations of the absorbed dose and energy dependence of plastic scintillators," Med. Phys. 32, 1265-1269 (2005).

12. Clift, M. A., Sutton, R. A., and Webb, D. V., "Water equivalence of plastic organic scintillators in megavoltage radiotherapy bremsstrahlung beams," Phys. Med. Biol. 45, 1885-1895 (2000).

13. Attix, F. H., "Energy imparted, energy transferred and net energy transferred," Phys. Med. Biol. 28, 1385-1390 $(1983)$.

14. X-5 Monte Carlo Team, "MCNP - A general Monte Carlo N-particle transport code, Version 5," Report LA-UR03-1987, Los Alamos National Laboratory, Los Alamos, NM, USA (2005).

15. McCarrick, J. F., "A numerical study of on-axis dose rate from Ta and W bremsstrahlung converter targets," Report UCRL-TR-212244, Lawrence Livermore national Laboratory, Livermore, CA, USA (2005).

16. Hubbell, J. H., "Photon mass attenuation and energy absorption coefficients from $1 \mathrm{keV}$ to $20 \mathrm{MeV}$," J. App. Rad. Iso. 33, 1269-1290 (1986).

17. http://www.eljentechnology.com/products.html

18. http://www.acqiris.com/products/digitizers/10-bit-cpci-6u-digitizers/dc282.html

19. Kudomi, N., "Energy calibration of plastic scintillators for low energy electrons by using Compton scatterings of $\gamma$ rays," Nucl. Inst. Meth. Phys. Res. A 430, 96-99 (1999).

20. Christiansen, B. H., "Commissioning of the new health physics instrumentation laboratory at the INEEL," Health Phys. 84, S168 (2003). 